\title{
Use of direct oral anticoagulants for acute pulmonary embolisms in obesity: a propensity-matched, multicentre case-control study
}

\begin{abstract}
To the Editor:
Copyright @The authors 2021

This version is distributed under the terms of the Creative Commons Attribution NonCommercial Licence 4.0. For commercial reproduction rights and permissions contact permissions@ersnet.org

Received: 26 March 2021 Accepted: 27 June 2021

\section{○@®®}

Direct oral anticoagulants (DOACs) are widely used as first-line treatment for pulmonary embolism (PE) in patients without contraindications [1]; however, limited data exists on the efficacy and safety in obesity. The most recent International Society of Thrombosis and Haemostasis guidelines recommend avoiding DOACs in individuals with body mass index (BMI) $>40 \mathrm{~kg} \cdot \mathrm{m}^{-2}$ or body weight $>120 \mathrm{~kg}$, due to lack of robust clinical efficacy data. Obtaining serum drug levels for therapeutic monitoring in this population has been suggested; however, testing of DOAC levels is neither widely available nor well validated in real-world clinical settings [2]. Given the ongoing uncertainty regarding the clinical outcomes with DOACs in treating acute PE in obese patients, this study aimed to evaluate the efficacy and safety of DOACs compared to warfarin in this population.

We conducted a multisite propensity scores matched case-control study combining databases from three hospitals in Queensland, Australia. The venous thromboembolism (VTE) database at Princess Alexandra Hospital (January 2015 to January 2020) and Patient Admission Records databases from Logan Hospital (January 2018 to January 2020) and Sunshine Coast University Hospital (January 2018 to January 2020) were screened to identify consecutive patients admitted with acute PE. Patients discharged directly from the emergency department were excluded as follow-up data was less reliable. Patients aged $>18$ years who had a BMI $>30 \mathrm{~kg} \cdot \mathrm{m}^{-2}$ and who were initiated on any oral anticoagulant (DOACs or warfarin) during the hospital admission were eligible. Patients who had other indications for anticoagulation or recurrent VTE were excluded. Demographic, clinical characteristics at diagnosis and outcome data were collected for eligible patients from the state-wide Electronic Medical Record. Ethical approval for this study was granted by the local Research Ethics Committee (HREC/2019/QMS/57882).

The primary outcome was the rate of recurrent VTE within 6 months of commencing anticoagulation, confirmed on diagnostic imaging. Secondary outcomes included: 1) rate of major, clinically relevant non-major and minor bleeding as defined by the International Society of Thrombosis and Haemostasis guidelines [3]; 2) all-cause mortality at 30 days and 6 months following the PE; and 3) length of hospital stay.

Propensity scores were generated for eligible patients using a logistical regression model with pre-specified variables. Propensity score analysis is a statistical method used to control for selection bias in observational studies [4]. Anticoagulation type (DOAC versus warfarin) was used as the dependent variable and the covariates included age, sex, PE severity index (PESI) score, BMI, estimated glomerular filtration rate (eGFR) and raised cardiac troponin. Propensity scores were matched to generate a DOAC group and a warfarin group at a 2:1 ratio using caliper width equal to 0.3 of the standard deviation of the propensity score. It was estimated that a target sample size of 58 patients per group were required, with rate of VTE recurrence of $5 \%$ at 6 months in both groups, and a clinically significant difference set at $10 \%$ (80\% power and $5 \%$ significance), using the principles of two-sample equivalence tests for proportions [5]. Outcome measures were determined on an intention-to-treat basis. The non-parametric continuous variables were

\footnotetext{
Shareable abstract (@ERSpublications)

Assessment of efficacy and safety of DOACs in treatment of pulmonary embolisms in obese patients provides reassurance that treatment with DOACs carries similar rates of recurrent VTE and
} bleeding complications to warfarin https://bit.ly/2VdrSXX

Cite this article as: Samaranayake CB, Keir G, Slader SAA, et al. Use of direct oral anticoagulants for acute pulmonary embolisms in obesity: a propensity-matched, multicentre case-control study. ERJ Open Res 2021; 7: 00379-2021 [DOI: 10.1183/23120541.00379-2021].


TABLE 1 Baseline characteristics and outcomes of study participants

\begin{tabular}{|c|c|c|c|}
\hline Characteristics & DOAC $^{\#}$ & Warfarin & $\mathrm{p}$-value \\
\hline \multicolumn{4}{|l|}{ Baseline characteristics } \\
\hline \multicolumn{4}{|l|}{ Demographics } \\
\hline Age years, median (interquartile range) & $60.1(36.2-84.1)$ & $59.0(35.4-82.3)$ & 0.83 \\
\hline Female, $n(\%)$ & $83(53.9)$ & $41(53.2)$ & 0.92 \\
\hline \multicolumn{4}{|l|}{ Anthropometrics } \\
\hline Height $\mathrm{cm}$, mean \pm sD & $170.9 \pm 9.3$ & $170.8 \pm 9.0$ & 0.91 \\
\hline Weight kg, mean \pm sD & $122.4 \pm 22.6$ & $125.9 \pm 33.5$ & 0.82 \\
\hline $\mathrm{BMI} \mathrm{kg} \cdot \mathrm{m}^{-2}$, mean $\pm \mathrm{SD}$ & $42.0 \pm 7.5$ & $43.3 \pm 11.8$ & 0.74 \\
\hline $\mathrm{BMI}>40 \mathrm{~kg} \cdot \mathrm{m}^{-2}$ or weight $>120 \mathrm{~kg}, \mathrm{n}(\%)$ & $104(67.5)$ & $46(59.7)$ & 0.24 \\
\hline \multicolumn{4}{|l|}{ Clinical parameters on presentation } \\
\hline First recorded SBP mmHg, mean \pm SD & $128.1 \pm 21.5$ & $124.5 \pm 25.7$ & 0.31 \\
\hline First recorded heart rate, mean \pm sD & $96.5 \pm 19.1$ & $96.6 \pm 18.1$ & 0.89 \\
\hline $\mathrm{S}_{\mathrm{pO}_{2}}<94 \%$ on presentation, $\mathrm{n}(\%)$ & $73(47.4)$ & $40(51.9)$ & 0.51 \\
\hline History of malignancy, $\mathrm{n}(\%)$ & $16(10.4)$ & $6(7.8)$ & 0.43 \\
\hline PESI score, mean \pm SD & $86.1 \pm 29.9$ & $88.7 \pm 33.7$ & 0.56 \\
\hline \multicolumn{4}{|l|}{ PESI score, $n(\%)$} \\
\hline Class V: very high risk & $14(9.1)$ & $7(9.1)$ & \\
\hline Class IV: high risk & $19(12.3)$ & $11(14.3)$ & 0.24 \\
\hline Class III: moderate risk & $49(31.8)$ & $24(31.2)$ & 0.89 \\
\hline Class II: low risk & $29(18.8)$ & $15(19.5)$ & 0.78 \\
\hline Class I: very low risk & $43(27.9)$ & $20(26.0)$ & 0.67 \\
\hline \multicolumn{4}{|l|}{ CTPA characteristics } \\
\hline \multicolumn{4}{|l|}{ Most proximal clot location, $\mathrm{n}(\%)$} \\
\hline Saddle/main PA & $36(23.4)$ & $13(16.7)$ & 0.47 \\
\hline Lobar arteries & $66(42.9)$ & $36(46.8)$ & 0.78 \\
\hline Segmental & $25(32.5)$ & $46(30.0)$ & 0.81 \\
\hline Clot number: bilateral & $133(86.3)$ & $62(80.5)$ & 0.50 \\
\hline RHS on CTPA, $n(\%)$ & $97(63.3)$ & $51(66.2)$ & 0.83 \\
\hline \multicolumn{4}{|l|}{ Echocardiographic parameters } \\
\hline LV impairment, $\mathrm{n}(\%)$ & $16(12.7)$ & $7(11.3)$ & 0.76 \\
\hline RV impairment, $\mathrm{n}(\%)$ & $78(61.9)$ & $39(60.0)$ & 0.89 \\
\hline RV dilatation, $\mathrm{n}(\%)$ & $92(73.1)$ & $41(66.1)$ & 0.46 \\
\hline RVSP $\mathrm{mmHg}$, mean \pm SD & $43.5 \pm 12.4$ & $43.9(10.5)$ & 0.85 \\
\hline \multicolumn{4}{|l|}{ Laboratory markers } \\
\hline Elevated troponin, $\mathrm{n}(\%)$ & $72(62.6)$ & $29(64.4)$ & 0.88 \\
\hline Lactate on presentation $\mathrm{mmol} \cdot \mathrm{L}^{-1}$, mean $\pm \mathrm{SD}$ & $2.3 \pm 1.4$ & $2.5 \pm 1.6$ & 0.52 \\
\hline eGFR $\mathrm{mL} \cdot \mathrm{min}^{-1} \cdot 1.73 \mathrm{~m}^{-2}$, mean \pm SD & $68.1 \pm 15.5$ & $67.3 \pm 20.2$ & 0.78 \\
\hline \multicolumn{4}{|l|}{ Pulmonary embolism risk category } \\
\hline Low risk & $39(25.3)$ & $16(20.8)$ & 0.45 \\
\hline Intermediate-low risk & $45(29.2)$ & $30(39.0)$ & 0.14 \\
\hline Intermediate-high risk & $57(37.0)$ & $22(28.6)$ & 0.24 \\
\hline High risk & $13(8.4)$ & $9(11.7)$ & 0.48 \\
\hline \multicolumn{4}{|l|}{ Initial treatment } \\
\hline Intravenous heparin infusion, $\mathrm{n}(\%)$ & $66(42.9)$ & $40(51.9)$ & 0.11 \\
\hline Low molecular-weight heparin, $\mathrm{n}(\%)$ & $53(34.4)$ & $28(36.4)$ & 0.69 \\
\hline Thrombolysis and heparin, $\mathrm{n}(\%)$ & $13(8.4)$ & $9(11.7)$ & 0.62 \\
\hline Upfront DOAC & $22(14.3)$ & & \\
\hline \multicolumn{4}{|l|}{ Type of DOAC and maintenance dose } \\
\hline Rivaroxaban 20 mg daily, n (\%) & $141(91.6)$ & & \\
\hline Apixaban 5 mg twice daily, $\mathrm{n}(\%)$ & $13(8.4)$ & & \\
\hline \multicolumn{4}{|l|}{ Outcomes at follow-up } \\
\hline \multicolumn{4}{|l|}{ Recurrent VTE within 6 months, $\mathrm{n}(\%)$} \\
\hline All recurrent VTE, n (\%) & $9(5.8)$ & $5(6.5)$ & 0.85 \\
\hline Recurrent pulmonary embolism, $\mathrm{n}(\%)$ & $4(2.6)$ & $3(3.9)$ & 0.59 \\
\hline \multicolumn{4}{|l|}{ Bleeding within 6 months } \\
\hline Major bleeding, $\mathrm{n}(\%)$ & $1(0.6)$ & $2(2.6)$ & 0.25 \\
\hline CRNM bleeding, n (\%) & $7(4.5)$ & $6(7.8)$ & 0.22 \\
\hline Minor bleeding, $\mathrm{n}(\%)$ & $14(9.1)$ & $5(6.5)$ & 0.58 \\
\hline
\end{tabular}




\begin{tabular}{|c|c|c|c|}
\hline Characteristics & DOAC & Warfarin & p-value \\
\hline \multicolumn{4}{|l|}{ Mortality } \\
\hline 30-day all-cause mortality, n (\%) & $2(1.3)$ & $3(3.9)$ & 0.22 \\
\hline 6-month all-cause mortality, n (\%) & $4(2.6)$ & $5(6.5)$ & 0.16 \\
\hline Length of hospital stay days, mean $\pm \mathrm{sD}$ & $5.1(3.8)$ & $7.9(4.1)$ & 0.01 \\
\hline \multicolumn{4}{|c|}{ 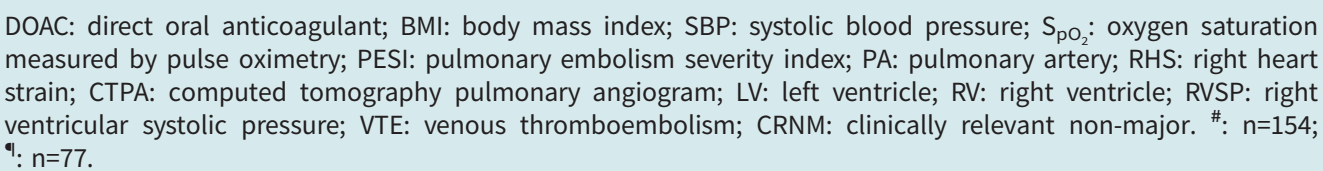 } \\
\hline
\end{tabular}

compared between the two groups using the Mann-Whitney test. The analysis was performed on SPSS Statistics V27 with the Python based extensions Fuzzy and PSM (IBM Corp.).

There were 1682 hospital presentations with acute PE during the study period across the three sites, and 848 were discharged directly from the emergency department. A total of 271 adult obese patients with a median (interquartile) age of 59 (41-78) years met inclusion criteria, and 231 underwent propensity score matching to the DOAC group $(n=154)$ and warfarin group $(n=77)$. No appropriate match was found for 40 patients. The mean (range) body weight of the study group was 123 (87-235) kg. The study population included 150 (64.9\%) patients who were morbidly obese (BMI $>40 \mathrm{~kg} \cdot \mathrm{m}^{-2}$ or body weight $>120 \mathrm{~kg}$ ), of which $36(15.6 \%)$ patients had a body weight $>160 \mathrm{~kg}$. The two groups were well matched at baseline (table 1). The intended duration of anticoagulation was $\geqslant 6$ months in all patients. Nine patients were lost to follow-up: seven $(4.5 \%)$ in the DOAC group and two $(2.6 \%)$ in the warfarin group.

On intention-to-treat analysis, recurrent VTE at 6 months occurred in 5.8\% $(n=9)$ of patients in the DOAC group compared to $6.6 \%(n=5)$ in the warfarin group (OR 0.89 (95\% CI 0.3-2.8), $\mathrm{p}=0.85$ ). The sensitivity analysis excluding patients lost to follow-up or deceased within the study period showed similar rates of recurrent VTE (6.3\% in DOAC group versus 7.1\% in warfarin group; OR 0.90 (95\% CI 0.3-2.7)). The overall rate of bleeding events was $14.3 \%(n=22)$ in the DOAC group and $16.9 \%(n=13)$ in the warfarin group (OR 0.82 (95\% CI 0.4-1.7), p=0.60). The rate of 30-day mortality was $1.3 \%(n=2)$ in the DOAC group compared to $3.9 \%(n=3)$ in the warfarin group (OR 0.32 (95\% CI $0.1-2.0), p=0.22)$. Amongst the patients who had a BMI $>40 \mathrm{~kg} \cdot \mathrm{m}^{-2}$ or body weight $>120 \mathrm{~kg}$, the rate of recurrent VTE was $5.8 \%(\mathrm{n}=6)$ in the DOAC group compared to $6.8 \%(\mathrm{n}=3)$ in the warfarin group, and bleeding events occurred in $9.6 \%$ $(n=10)$ in the DOAC group compared to $15.2 \%(n=7)$ in the warfarin group. In patients who had intermediate or high-risk PE, the rate of recurrent VTE was $4.3 \%(n=5)$ in the DOAC group compared to $6.6 \%(n=4)$ in the warfarin group.

This case-control study assessed outcomes at 6 months in obese patients with acute PE requiring hospital admission who received treatment with DOACs compared to warfarin. Treatment with DOACs carries similar rates of recurrent VTE and overall bleeding complications compared to warfarin. As none of the patients in the DOAC group had treatment adjustments based on therapeutic drug monitoring, our findings are directly applicable to real-world clinical settings where serum DOAC level measurements may not be routinely available.

Major bleeding events were rare and clinically relevant non-major bleeding events were similar between the two groups. Most of these bleeding events occurred within the first 2 months of discharge from hospital. Previous studies comparing DOACs to warfarin have indicated a trend towards lower bleeding with DOACs, and is evident in patients who have both normal and elevated BMI [6-8]. However, there is also evidence indicating that each DOAC may have differences in outcomes when used in obese patients [9], and studies comparing different DOACs in this patient population are needed. An increased average length of hospital stay following PE was observed in the warfarin group (7.9 days versus 5.1 days in the DOAC group, $\mathrm{p}=0.01$ ), mostly due to patients awaiting therapeutic INR levels prior to discharge. The longer length of hospital stay in patients initiated on warfarin for VTE compared to DOACs has been shown in previous studies [10,11], and is associated with higher healthcare costs [12].

A strength of our study is the propensity score matched study groups, which reduces bias associated with the case-controlled design. Furthermore, the patients were well phenotyped at baseline and extensively risk 
stratified. Our results are concordant with the findings of a recent meta-analysis combining observational studies on DOACs for treatment of VTE [7]. Coons et al. [13] also reported similar recurrent VTE rates of $6.5 \%$ with DOACs compared to $6.4 \%$ with warfarin at 12 months in patients with a body weight $>100 \mathrm{~kg}$ who had any form of VTE. In a large registry based retrospective study of obese patients with all forms of VTE, Spyropoulos et al. [12] also showed similar efficacy between rivaroxaban and warfarin with recurrent VTE rates of $16.8 \%$ and $15.9 \%$ at 12 months retrospectively. Recent evidence also suggests that's DOACs may have similar risks of long-term complications including chronic thromboembolic pulmonary hypertension compared to warfarin in morbidly obese patients with intermediate or high-risk PE [14].

This study has several limitations. INR monitoring and warfarin dose adjustments were carried out in the community in line with routine clinical practice, and we were therefore unable to account for the effect of sub- or supratherapeutic INR on outcome measures. The number of patients who received apixaban was very small, therefore meaningful comparison of DOAC subtypes could not be performed. As a majority of the patients received rivaroxaban, the applicability of the findings to other DOACs is limited. The impact of different initial high dose administration durations (3 weeks for rivaroxaban and 7 days for apixaban) may be an important consideration in this patient group in the event of potential underdosing related to bodyweight. The study excluded patients discharged directly from the emergency department, which may impact on generalisability of the results, particularly in patients with lower risk PE. Few patients in our study had extreme obesity, and the management of such patients (e.g. body weight $>160 \mathrm{~kg}$ ) requires further research.

In summary, our study adds to the emerging evidence regarding the efficacy and safety of treating PE with DOACs in obese patients in a real-world clinical setting without anti-factor-Xa or serum DOAC level measurements guiding treatment. The rate of recurrent VTE and bleeding complications at 6 months were similar between DOACs and warfarin. Larger studies are needed to assess for differences in outcome according to subtype of DOAC in this patient population.

Chinthaka B. Samaranayake $\oplus^{1}$, Gregory Keir ${ }^{1,2}$, Simon A.A. Slader ${ }^{2}$, Tommy Tseng ${ }^{2}$, Khoa Tran ${ }^{1,3}$, James Anderson ${ }^{4,5}$, Andrew McCann ${ }^{2}$, Colm McCabe ${ }^{6,7}$ and John W. Upham ${ }^{1,2}$

${ }^{1}$ Faculty of Medicine, University of Queensland, Brisbane, Australia. ${ }^{2}$ Princess Alexandra Hospital, Brisbane, Australia. ${ }^{3}$ Logan Hospital, Brisbane, Australia. ${ }^{4}$ Sunshine Coast University Hospital, Birtinya, Australia. ${ }^{5}$ School of Medicine, Griffith University, Southport, Australia. ${ }^{6}$ Royal Brompton and Harefield National Health Service Trust, London, UK. ${ }^{7}$ National Heart and Lung Institute, Imperial College, London, UK.

Corresponding author: Chinthaka B. Samaranayake (c.samaranayake@uq.edu.au)

Acknowledgements: The authors would like to thank Roney Neale, Clinical Nurse Consultant from the Vascular Medicine Dept at Princess Alexandra Hospital for maintaining the Venous Thromboembolism Database.

Provenance: Submitted article, peer reviewed.

Conflict of interest: None declared.

Support statement: C.B. Samaranayake is supported by a National Health and Medical Research Council Australia Postgraduate Scholarship.

References

1 Konstantinides SV, Meyer G, Becattini C, et al. 2019 ESC Guidelines for the diagnosis and management of acute pulmonary embolism developed in collaboration with the European Respiratory Society (ERS). Eur Heart J 2020; 41: 543-603.

2 Patel JP, Byrne RA, Patel RK, et al. Progress in the monitoring of direct oral anticoagulant therapy. $\mathrm{Br} J$ Haematol 2019; 184: 912-924.

3 Schulman S, Kearon C. Definition of major bleeding in clinical investigations of antihemostatic medicinal products in non-surgical patients. J Thromb Haemost 2005; 3: 692-694.

4 Austin PC. An introduction to propensity score methods for reducing the effects of confounding in observational studies. Multivariate Behav Res 2011; 46: 399-424. 
5 Chow SC, Shao J, Wang H. Sample Size Calculations in Clinical Research. 2nd Edn. New York, Chapman and Hall/CRC, 2007.

6 van der Hulle T, Kooiman J, den Exter PL, et al. Effectiveness and safety of novel oral anticoagulants as compared with vitamin $\mathrm{K}$ antagonists in the treatment of acute symptomatic venous thromboembolism: a systematic review and meta-analysis. J Thromb Haemost 2014; 12: 320-328.

7 Elshafei MN, Mohamed MFH, El-Bardissy A, et al. Comparative effectiveness and safety of direct oral anticoagulants compared to warfarin in morbidly obese patients with acute venous thromboembolism: systematic review and a meta-analysis. J Thromb Thrombolysis 2021; 51: 388-396.

8 López-López JA, Sterne JAC, Thom HHZ, et al. Oral anticoagulants for prevention of stroke in atrial fibrillation: systematic review, network meta-analysis, and cost effectiveness analysis. BMJ 2017; 359: j5058.

9 Sebaaly J, Kelley D. Direct oral anticoagulants in obesity: an updated literature review. Ann Pharmacother 2020; 54: 1144-1158.

10 Saint CA, Castelli MR, Crannage AJ, et al. Comparison of hospital length of stay in patients treated with non-vitamin $\mathrm{K}$ oral anticoagulants or parenteral agents plus warfarin for venous thromboembolism. SAGE Open Med 2017; 5: 2050312117719628.

11 Badreldin $\mathrm{H}$. Hospital length of stay in patients initiated on direct oral anticoagulants versus warfarin for venous thromboembolism: a real-world single-center study. J Thromb Thrombolysis 2018; 46: 16-21.

12 Spyropoulos AC, Ashton V, Chen YW, et al. Rivaroxaban versus warfarin treatment among morbidly obese patients with venous thromboembolism: comparative effectiveness, safety, and costs. Thromb Res 2019; 182: 159-166.

13 Coons JC, Albert L, Bejjani A, et al. Effectiveness and safety of direct oral anticoagulants versus warfarin in obese patients with acute venous thromboembolism. Pharmacotherapy 2020; 40: 204-210.

14 Lachant DJ, Bach C, Fe A, et al. Direct oral anticoagulant therapy in patients with morbid obesity after intermediate- or high-risk pulmonary emboli. ERJ Open Res 2021; 7: 00554-2020. 